Article

\title{
Selenoprotein-Transgenic Chlamydomonas reinhardtii
}

Qintang Hou ${ }^{1}$, Shi Qiu ${ }^{2}$, Qiong Liu ${ }^{2, *}$, Jing Tian ${ }^{1}$, Zhangli Hu ${ }^{1}$ and Jiazuan Ni ${ }^{1, *}$

1 Shenzhen Key Laboratory of Marine Biotechnology and Ecology, Department of Marine Biology, Shenzhen University, Shenzhen 518060, China; E-Mails: hqtdream2007@sina.com (Q.H.); jing.tianjingtj@gmail.com (J.T.); huzl@szu.edu.cn (Z.H.)

2 Shenzhen Key Laboratory of Microbial Genetic Engineering, College of Life Sciences, Shenzhen University, Shenzhen 518060, China; E-Mail: qiuqiushi@hotmail.com

* Authors to whom correspondence should be addressed; E-Mails: liuqiong@szu.edu.cn (Q.L.); jzni@szu.edu.cn (J.N.); Tel.: +86-755-26535432; Fax: +86-755-26534274.

Received: 29 December 2012; in revised form: 4 February 2013 / Accepted: 13 February 2013 / Published: 26 February 2013

\begin{abstract}
Selenium (Se) deficiency is associated with the occurrence of many diseases. However, excessive Se supplementation, especially with inorganic Se, can result in toxicity. Selenoproteins are the major forms of Se in vivo to exert its biological function. Expression of those selenoproteins, especially with the application of a newly developed system, is thus very important for studying the mechanism of Se in nutrition. The use of Chlamydomonas reinhardtii ( $C$. reinhardtii) as a biological vector to express an heterogeneous protein is still at the initial stages of development. In order to investigate the possibility of using this system to express selenoproteins, human 15-KDa selenoprotein (Sep15), a small but widely distributed selenoprotein in mammals, was chosen for the expression platform test. Apart from the wild-type human Sep15 gene fragment, two Sep15 recombinants were constructed containing Sep15 open reading frame (ORF) and the selenocysteine insertion sequence (SECIS) element from either human Sep15 or C. reinhardtii selenoprotein W1, a highly expressed selenoprotein in this alga. Those Sep15-containing plasmids were transformed into $C$. reinhardtii CC-849 cells. Results showed that Sep15 fragments were successfully inserted into the nuclear genome and expressed Sep15 protein in the cells. The transgenic and wild-type algae demonstrated similar growth curves in low Se culture medium. To our knowledge, this is the first report on expressing human selenoprotein in green alga.
\end{abstract}


Keywords: $15-\mathrm{KDa}$ selenoprotein (Sep15); alga; Chlamydomonas reinhardtii (C. reinhardtii); selenium (Se); selenocysteine insertion sequence (SECIS)

\section{Introduction}

Selenium (Se) deficiency is associated with the occurrence of many diseases, such as cancer, cardiovascular disease, and Alzheimer's disease [1-3]. Se exerts its biological functions mainly through selenoproteins [1,4]. The $15 \mathrm{KDa}$ selenoprotein (Sep15) is one of the key selenoproteins modulating in vivo redox balance and participating in the formation of disulfide bonds during protein folding [5]. In the selenoprotein family, Sep15 is relatively small and widely distributed in most mammalian tissues [1], but its structure and function remain unclear. Thus, this protein was chosen for the test of selenoprotein expression in a new system - C. reinhardtii.

Resembling other selenoproteins, Sep15 has a special gene structure, wherein the active selenocysteine ( $\mathrm{Sec}$ ) is encoded by a traditional stop codon UGA in the open reading frame (ORF). In order to decode the in-frame UGA to a Sec residue, a special stem-loop structure, designated as Sec insertion sequence (SECIS) element, must be present in the $3^{\prime}$-untranslated region (UTR) of a eukaryotic selenoprotein gene to guide the Sec translation and incorporation into the protein [6,7].

It is known that yeast and land plants do not express selenoproteins [8]. Surprisingly, a variety of selenoproteins were found in algae such as Ostreococcus tauri, Ostreococcus lucimarinus, and C. reinhardtii [8-10]. C. reinhardtii was reported to contain 10 selenoproteins [11], including two Selenoprotein W homologs (SelW1, SelW2), two phospholipid hydroperoxide glutathione peroxidase homologs (PHGPx1, PHGPx2), two selenoprotein M homologs (SelM1, SelM2), selenoprotein T (SelT1), selenoprotein K (SelK1), thioredoxin reductase (TR1), and methionine sulfoxide reductase A (MsrA1). Among those selenoproteins, SelW1 and PHGPx1 are two highly expressed proteins in the C. reinhardtii. Those $C$. reinhardtii selenoprotein genes share common origins with their mammalian homologs [8]. Additionally, $C$. reinhardtii cells contain a Sec tRNA that specifically recognizes the UGA codon. Thus, it is reasonable to detect the possibility for $C$. reinhardtii to use the endogenous selenoprotein expressing system, including Sec tRNA, SECIS element and some trans-acting factors, to express heterogeneous selenoproteins.

Currently, the use of expressing exogenous protein in $C$. reinhardtii is only at the initial research stage. This expression system has the advantages of non-toxic, low cost and short growth cycle compared with other systems. Several proteins have been already expressed successfully in C. reinhardtii through either nuclear transformation or chloroplast transformation $[12,13]$, which provide references for our present study. Hereby three forms of human Sep15 gene fragments were constructed and expressed in C. reinhardtii. To our knowledge, this is the first report about expressing a human selenoprotein in green alga. 


\section{Experimental Section}

\subsection{Materials}

A cell-wall-deficient $C$. reinhardtii strain CC-849 was obtained from the Chlamydomonas Genetic Center of Duke University (Durham, NC, USA). The expression vector pH124 containing the Amp ${ }^{+}$ and zeocin resistance was self-stored in the Institute of Marine Biotechnology, Shenzhen University. The E. coli strain DH5 $\alpha$ was preserved in our laboratory. pMD18-T vector and ExTaq enzyme were purchased from Takara (Dalian, China). Plasmid pMD18-T-Sep15 containing a human Sep15 gene was constructed previously in our laboratory. Rabbit polyclonal antibody against the $N$-terminal peptide of Sep15 was synthesized by Abmart (Shanghai, China). Pierce ECL detection kit for Western blot analysis was purchased from Thermo Fisher (Rockford, USA).

Restriction enzymes, T4 ligase, DNA polymerase, plasmid purification kit, DNA purification kit, DNA gel extraction kit, RT-PCR reaction kit were purchased from Takara (Dalian, China). Genome extraction kit was purchased from Sbsgene (Shanghai, China). PCR primer syntheses and DNA sequencing were performed by Sangon Biotech (Shanghai, China). TRIzol reagent was from Invitrogen (Shanghai, China). All chemical reagents, except otherwise noted, were obtained from HS-Science (Shenzhen, China). Chemicals like hydrochloric acid, nitric acid, perchloric acid, potassium hydroxide, and potassium borohydride were superior grade of pure (GR), while others including sodium selenite were of analytical pure grade (AR).

\subsection{Primer Design}

The sequences of human Sep15 and C. reinhardtii SelW1 genes were extracted from the NCBI database and analyzed for their SECIS elements with the online program SECISearch. Primers listed in Table 1 were designed by Primer 5.0 according to the published sequences. Two forward primers $F_{1}$, $F_{2}$ and reverse primers $R_{1}, R_{2}$ were designed to amplify the wild-type full-length gene (containing both ORF and SECIS), the ORF, and the SECIS element of Sep15, respectively. The primers $F_{w}$ and $R_{w}$ were designed to amplify the SECIS of $C$. reinhardtii SelW1, and primers $F_{\text {ble }}$ and $\mathrm{R}_{\text {ble }}$ were used for the amplification of ble gene. The introduction of Clal site and protection bases in both ORF and SECIS serves as an overlap sequence for constructing the human Sep15 recombinant.

Table 1. Primers designed in the paper ${ }^{\text {a }}$.

\begin{tabular}{cc}
\hline name & primer sequence \\
\hline $\mathrm{F}_{1}$ & 5'-CTAGCTAGCATGCATCATCATCATCATCATGTGTCTGCTTTT GGGGCAGAG-3' \\
$\mathrm{R}_{1}$ & 5'-CCATCGATG GAAAAGGATAGGACAAAATTTAAGCA-3' \\
$\mathrm{F}_{2}$ & 5'-CCATCGATG GTACATAAAAACTTTGTAGCTTCATT-3' \\
$\mathrm{R}_{2}$ & 5'-ACGCGTCGAC ATCACTTTTAAATGGACTTTTCTGT-3' \\
$\mathrm{F}_{\mathrm{W}}$ & 5'-CCATCGATG GACTGAGCACTGCCGCCCTGT-3' \\
$\mathrm{R}_{\mathrm{W}}$ & 5'-ACGCGTCGAC GGCACAGCCTCATGACCTCCTA-3' \\
$\mathrm{F}_{\mathrm{ble}}$ & 5'-GGCCAAGCTGACCAGCGCCGTTC-3' \\
$\mathrm{R}_{\mathrm{ble}}$ & 5'-CTCCCGCCCCCACGGCTGCTC-3' \\
\hline
\end{tabular}

${ }^{a}$ protection bases are in bold type. Restriction enzyme cutting sites are underlined including Nhe I (GCTAGC), Cla I ( $\underline{\text { ATCGAT) }}$ and Sal I (GTCGAC). Start codon ATG is boxed off. 


\subsection{Plasmid Construction}

C. reinhardtii CC-849 cells were cultured in tris-acetate-phosphate (TAP) medium to the late exponential growth phase. Approximately $5 \times 10^{7}$ cells were harvested and washed twice with DEPC-treated $\mathrm{H}_{2} \mathrm{O}$. Total RNA was extracted by Trizol reagent. The first-strand cDNA was reversed-transcripted using oligo-dT primers and ReverTra Ace reverse transcriptase (Toyobo, Japan) at $42{ }^{\circ} \mathrm{C}$ for $20 \mathrm{~min}, 99^{\circ} \mathrm{C}$ for $5 \mathrm{~min}$, and $4{ }^{\circ} \mathrm{C}$ for $5 \mathrm{~min}$. The cDNA was used as a template to amplify the SECIS element of $C$. reinhardtii SelW1 with the primers $F_{w}$ and $\mathrm{R}_{\mathrm{w}}$ under the following PCR conditions: 30 cycles of denaturation at $94{ }^{\circ} \mathrm{C}$ for $30 \mathrm{~s}$, annealing at $55{ }^{\circ} \mathrm{C}$ for $30 \mathrm{~s}$ and extension at $72{ }^{\circ} \mathrm{C}$ for $40 \mathrm{~s}$.

Plasmid pMD18-T-Sep15 containing human Sep15 gene was used as a template for PCR amplification of the full-length Sep15 (primers $F_{1}, R_{2}$ ), its ORF (primers $F_{1}, R_{1}$ ), and its SECIS element (primers $\mathrm{F}_{2}, \mathrm{R}_{2}$ ). PCR was performed under the following reaction conditions: preheating at $94{ }^{\circ} \mathrm{C}$ for $5 \mathrm{~min}, 25$ cycles of circulation at $94{ }^{\circ} \mathrm{C}$ for $30 \mathrm{~s}, 59^{\circ} \mathrm{C}$ for $30 \mathrm{~s}$, and $72{ }^{\circ} \mathrm{C}$ for $40 \mathrm{~s}$, extension at $72{ }^{\circ} \mathrm{C}$ for $7 \mathrm{~min}$.

The DNA fragments of Sep15 ORF and SECIS elements (from either C. reinhardtii SelW1 or human Sep15 genes) were used as templates and amplified by overlapping PCR to construct two recombinants of human Sep15 ORF plus $C$. reinhardtii SelW1 SECIS (primers $\mathrm{F}_{1}, \mathrm{R}_{\mathrm{w}}$ ) and human Sep15 ORF plus SECIS (primers $\mathrm{F}_{1}, \mathrm{R}_{2}$ ). PCR conditions: preheating at $94{ }^{\circ} \mathrm{C}$ for $5 \mathrm{~min}$; 5 cycles of circulation at $94{ }^{\circ} \mathrm{C}$ for $30 \mathrm{~s}, 42^{\circ} \mathrm{C} 30 \mathrm{~s}$, and $72{ }^{\circ} \mathrm{C} 40 \mathrm{~s}$; addition of each pair of primers and running another 20 cycles of circulation at $94{ }^{\circ} \mathrm{C}$ for $30 \mathrm{~s}, 55{ }^{\circ} \mathrm{C} 30 \mathrm{~s}$, and $72{ }^{\circ} \mathrm{C} 40 \mathrm{~s}$; extension at $72{ }^{\circ} \mathrm{C}$ for $7 \mathrm{~min}$.

Three PCR products of wild-type and two recombinant Sep15 fragments were inserted into the pMD18-T vector respectively, and transformed into $\mathrm{CaCl}_{2}$ competent E. coli (DH5 $\alpha$ strain) cells for ampicillin selection. The isolated plasmids were checked by PCR amplification and enzymatic digestion (with Nhe I and Sal I). Those Sep15-containing plasmids, including the human Sep15 ORF plus SECIS (pMD18-T-Sep15ORF-hSECIS), the human Sep15 ORF plus C. reinhardtii SelW1 SECIS (pMD18-T-Sep15ORF-chSECIS), and wild-type human Sep15 fragment (pMD18-T-wtSep15), were sequenced in Sangon Biotech (Shanghai, China).

Those sequence-confirmed Sep15-containing plasmids were then digested with Sal I restriction enzyme, purified, and the sticky ends were filled into blunt ones by two cycles of PCR using Pfu DNA polymerase without primers. After purification, the products were digested with Nhe I restriction enzyme to possess 5'-stick and 3'-blunt ends. The expression vector pH124 was then digested with the restriction enzymes Nhe I and PmaC I to get the same ends, so that those Sep15 fragments were inserted into the $\mathrm{pH} 124$ plasmids respectively. The constructed plasmids were transformed into E. coli cells, and PCR detection was performed to confirm the successful insertion. Three Sep15 expression plasmids were therefore constructed, including pH124-Sep15ORF-hSECIS, pH124-Sep15ORF-chSECIS, and pH124-wtSep15. 


\subsection{Nuclear Transformation of C. reinhardtii}

Monoclonal CC-849 cells were inoculated into sterile TAP liquid medium and cultured to mid-logarithmic phase (approximately $1-2 \times 10^{6}$ cells $/ \mathrm{mL}$ ) in the incubator under the following conditions: temperature, $25{ }^{\circ} \mathrm{C}$; brightness/darkness ratio, $16 / 8 \mathrm{~h}$; light intensity, $6000 \mathrm{l} \times$. In order to achieve high transformation efficiency, three Sep15 expression plasmids were Not I-digested into linear plasmids before they were transformed into CC-849 cells by the glass-bead method [13]. Briefly, CC-849 cells in the mid-log phase were harvested by centrifugation at $5000 \mathrm{rpm}$ for $5 \mathrm{~min}$, suspended with fresh TAP, and adjusted to $2 \times 10^{8}$ cells $/ \mathrm{mL}$. $300 \mu \mathrm{L}$ cell suspension was transferred into EP tubes containing 0.5-mm-diameter sterilized glass beads. 2-5 $\mu \mathrm{g}$ linearized Sep15-containing pH124 plasmids were then added. A "no DNA" control was set up. The cell/glass bead/DNA suspension was shaken in vortex for $18 \mathrm{~s}$ at top speed, transferred into $20 \mathrm{~mL}$ TAP medium, and cultured for about $25 \mathrm{~h}$ by shaking at $100 \mathrm{rpm}$. The cells were centrifuged down, gently resuspended in TAP medium, mixed with $0.5 \%$ molten agar in TAP (below $42{ }^{\circ} \mathrm{C}$, no antibiotic), and rapidly poured onto $2 \%$ TAP-agar plates containing $10 \mu \mathrm{g} / \mathrm{mL}$ zeocin. The plates were inverted and left under the light $(60001 \times)$ at $25^{\circ} \mathrm{C}$. Green colonies were visible after $2-3$ weeks.

\subsection{Identification of Human Sep15 Fragments in C. Reinhardtii}

The transgenic C. reinhardtii cells were harvested and genomic DNA was extracted for with the genomic extraction kit. PCR was performed using the genome as a template to check the presence of ble gene fragment (about $464 \mathrm{bp}$ ) with the primers $\mathrm{F}_{\text {ble }}$ and $\mathrm{R}_{\text {ble, }}$ or to check the presence of Sep15 fragments: primers $\mathrm{F}_{1}$ and $\mathrm{R}_{2}$ for wtSep15 (about $1164 \mathrm{bp}$ ) and Sep15ORF-hSECIS (about $681 \mathrm{bp}$ ), primers $\mathrm{F}_{1}$ and $\mathrm{R}_{\mathrm{w}}$ for Sep15ORF-chSECIS (about $690 \mathrm{bp}$ ). Reaction conditions: $95{ }^{\circ} \mathrm{C}$ for 5 min; $95{ }^{\circ} \mathrm{C}$ for $30 \mathrm{~s}, 55^{\circ} \mathrm{C}$ for $30 \mathrm{~s}, 72^{\circ} \mathrm{C}$ for $40 \mathrm{~s} ; 30$ cycles; $72{ }^{\circ} \mathrm{C}$ extension for $7 \mathrm{~min}$.

To analyze the mRNA expression level, the transgenic algae were cultured to mid-log phase, heat-shocked for $20 \mathrm{~min}$ at $42{ }^{\circ} \mathrm{C}$ to promote exogenous gene transcription, and then put back to the incubator to grow for another 1-2 h. Total RNA were extracted from the cells with TRIzol. RT-PCR was performed using the same pairs of primers described in the genomic DNA amplification for wtSep15, Sep15ORF-hSECIS, and Sep15ORF-chSECIS, respectively. RT conditions: $30^{\circ} \mathrm{C}, 10 \mathrm{~min}$; $42{ }^{\circ} \mathrm{C}, 30 \mathrm{~min} ; 99{ }^{\circ} \mathrm{C}, 5 \mathrm{~min} ; 4^{\circ} \mathrm{C}, 5 \mathrm{~min}$. PCR conditions: $94{ }^{\circ} \mathrm{C} 5 \mathrm{~min} ; 94{ }^{\circ} \mathrm{C} 1 \mathrm{~min}, 55{ }^{\circ} \mathrm{C} 30 \mathrm{~s}$, $72{ }^{\circ} \mathrm{C} 40 \mathrm{~s}, 30$ cycles; $72{ }^{\circ} \mathrm{C} 7 \mathrm{~min}$.

To analyze the expression of human Sep15 protein, the transgenic algae were grown in the medium supplemented with $\mathrm{Na}_{2} \mathrm{SeO}_{3}$ to a final concentration of $10 \mu \mathrm{mol} / \mathrm{L}$. Both the transgenic and wild-type C. reinhardtii cells were collected by centrifugation. Cold acetone was added to the cells and stored at $-20{ }^{\circ} \mathrm{C}$ overnight to remove the pigment. The cells were then centrifuged and washed twice with cold acetone. After complete evaporation of acetone on ice, the algal pellets were lysed in cell lysis solution by ultrasonication. After centrifugation, the supernatants were separated by SDS-PAGE and analyzed for Sep15 expression by Western blot using a rabbit polyclonal antibody against Sep15 (1:50 dilution). The immune complexes were revealed by enhanced chemiluminescence with the Pierce ECL detection kit. 


\subsection{Evaluation of Alga Growth}

Alga growth was evaluated by its cell density and dry-weight. The wild-type and transgenic C. reinhardtii were inoculated separately into $250 \mathrm{~mL}$ TAP medium containing $\mathrm{Na}_{2} \mathrm{SeO}_{3}$ at a series of final concentrations of $0,5,10,15$, and $20 \mu \mathrm{mol} / \mathrm{L}$, each in triplicates. Cell density was detected every $24 \mathrm{~h}$ by a spectrometer at $650 \mathrm{~nm}$ wave-length $\left(\mathrm{A}_{650}\right)$. To measure alga dry-weight, an aliquot of $50 \mathrm{~mL}$ cell culture was collected after growing for $24 \mathrm{~h}$, followed by a $20 \mathrm{~mL}$-collection at 48,72 , and $96 \mathrm{~h}$, respectively. Those aliquots of cell cultures were harvested at $5000 \mathrm{rpm}$ for $5 \mathrm{~min}$, transferred to pre-weighed EP tubes, freeze-dried to a constant weight, and weighed on an analytical balance. Data were expressed as the mean $\pm \mathrm{SD}$ of triplicate samples.

\subsection{Se Measurement}

Se in the algae was analyzed using the method of hydroxide generation-atomic fluorescence spectrometry (HG-AFS) [14,15]. Briefly, 0.02 g dry-algae was accurately weighed, digested with mixed acid $\left(\mathrm{HNO}_{3} / \mathrm{HClO}_{4}, \mathrm{v} / \mathrm{v}=4: 1\right)$, reduced by $50 \% \mathrm{HCl}$, filled with $5 \% \mathrm{HCl}$ solution to the final volume, and measured by an atomic fluorescence spectrometer (AFS-920, Jitian Instrument Co., Beijing, China). Instrument running condition: carrier solution, $5 \% \mathrm{HCl}$; reducing agent, $2 \% \mathrm{NaBH}_{4}$; lamp current, $80 \mathrm{~mA}$; negative voltage, $270 \mathrm{~V}$. Analytical pure sodium selenite was used to prepare a stock solution of $10 \mathrm{ng} / \mathrm{mL}$ Se. A series of standard solutions at 1, 2, 4, 6, 8 and $10 \mathrm{ng} / \mathrm{mL}$ were diluted automatically from the stock solution for calibration curve. Each sample was performed in independent triplicates. Statistical analysis was performed using statistical software SPSS 11.5. $P<0.05$ was considered as significant difference. Data were expressed as the mean $\pm \mathrm{SD}$ of triplicate samples.

\section{Results}

\subsection{Construction of Three Types of Human Sep15 Expression Plasmids}

RT-PCR was performed to amplify the SECIS element of C. reinhardtii SelW1 (219 bp). Full-length human Sep15 gene (1164 bp), together with its ORF (482 bp) and SECIS element (209 bp), were amplified from the self-constructed plasmid pMD18-T-Sep15. During PCR-amplification, an overlapping DNA sequence, containing a Cla I restriction site and several protection bases, was introduced into the 3'-end of Sep15 ORF and the 5'-ends of two SECIS elements, which facilitated the operation of overlap PCR to get the recombinants of Sep15ORF-hSECIS (681 bp) and Sep15ORF-chSECIS (690 bp). The full-length Sep15 (wild-type Sep15, wtSep15) and two Sep15 recombinants were then inserted respectively into the pMD18-T vector and transformed into E. coli DH5a. Plasmids isolated from the E. coli cells were checked by PCR amplification and restriction enzyme digestion (Figure 1) for the proper molecular sizes of three types of Sep15 inserts. Those Sep15 inserts were further confirmed by DNA sequencing to be $100 \%$ correct (shown in the Supplementary Data). The wtSep15 fragment contains the start codon ATG, Sec-decoding TGA, stop codon TAA, and SECIS element in the 3'-UTR, which are boxed off in the Supplementary Data. The sites of restriction enzymes, including Nhe I (GCTAGC), Cla I (ATCGAT) and Sal I (GTCGAC), are underlined. 
The wtSep15 and Sep15ORF-hSECIS fragments have the same ORFs and SECIS elements of human Sep15 gene, except the distance between the stop codon TAA and SECIS element. For the fragments of wtSep15, Sep15ORF-hSECIS and Sep15ORF-chSECIS, those distances between the stop codon and SECIS element are 574, 94 and $107 \mathrm{nt}$, respectively. The distances in the two recombinants are similar, which were designed according to that of $C$. reinhardtii SelW1 gene, in order to use the algal selenoprotein translation system to express human Sep15. Sequence analysis with the SECISearch program showed that the secondary structures of SECIS elements from the mRNAs of human Sep15 and $C$. reinhardtii SelW1 have the same conserved pattern AUGA_AA_GA, a characteristic of eukaryotic selenoprotein SECIS element. Such similarity lays the foundation for the exchange of two SECIS elements for heterogeneous expression of human Sep15 in C. reinhardtii.

Figure 1. Detection of three types of Sep15 fragments by plasmid PCR analysis (a) and enzymatic digestion $(\mathbf{b})$.

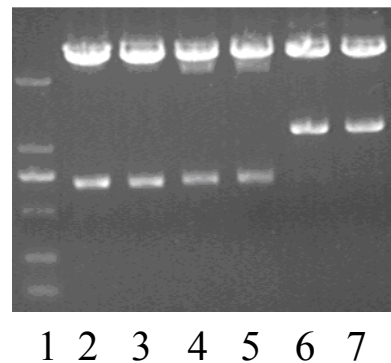

(a)



(b)

Lanes: 1: DL2000 marker (2000, 1000, 750, 500, 250, 100 bp); 2 \& 3: Sep15ORF-hSECIS; 4 \& 5: Sep15ORF-chSECIS; 6 \& 7: wtSep15.

The pH124 expression vector contains the multiple-promoter Hsp70-RBCS2, which can be heat-activated and light-induced for the expression of exogenous genes in C. reinhardtii. However, the presence of several Sal I cutting sites limits the usage of this enzyme for the vector. Thus, the obtained Sep15 fragments were firstly digested with Sal I, and then the sticky ends were filled, followed by further digestion with Nhe I to obtain the fragments containing 5'-sticky and 3'-flat ends. The expression vector pH124 was also cut with Nhe I and PmaC I to generate the same ends. The Sep15 fragments were then ligated with the $\mathrm{pH} 124$ plasmids, and transformed into competent $E$. coli cells. Successful insertion of each Sep15ORF-hSECIS, wtSep15, and Sep15ORF-chSECIS into the pH124 vector was detected by plasmid PCR.

\subsection{Identification of Human Sep15 in C. reinhardtii}

Plasmid-transformed algae were selected by the zeocin-containing plates due to the expression of ble gene constructed in the $\mathrm{pH} 124$ vector. CC-849 cells and the no-ble-plasmid transformed cells are very sensitive to zeocin that they could not grow on the zeocin-containing plate. Positively transformed cells, including pH124-wtSep15, pH124-Sep15ORF-hSECIS, pH124-Sep15ORF-chSECIS, and vacant pH124 transformed cells, expressed Ble protein to resist zeocin, leading to the growth of $C$. reinhardtii on zeocin-containing plates. 
To investigate if human Sep15 fragments had been inserted into the genomes of CC-849 cells, genomic DNAs were extracted from the monoclonal algae grown on the zeocin-containing plates and medium. Genomic PCR was performed to identify positive transformants via the detection of ble gene and the fragments of wt-Sep15, Sep15ORF-hSECIS, and Sep15ORF-chSECIS. As shown in Figure 2a, lanes 2-9 contained the ORF fragments (482 bp) amplified from the pH124-Sep15ORF-hSECIS transformed algae. Seven out of eight clones in the corresponding plates had their Sep15 ORFs integrated into the genomes of CC-849 cells. Lanes 10-17 contained the Sep15ORF-chSECIS fragments $(690 \mathrm{bp})$ amplified from the genomes of pH124-Sep15ORF-chSECIS transformed algae, and lanes 18-20 contained the Sep15 ORFs amplified from the pH124-wtSep15 transformed algae. All those clones had their Sep15 fragments positively integrated into the algal genomes.

Figure 2. Detection of human Sep15 in the transformed C. reinhardtii CC-849 cells by genomic PCR (a), RT-PCR (b), and Western blot (c) analyses.

(a)

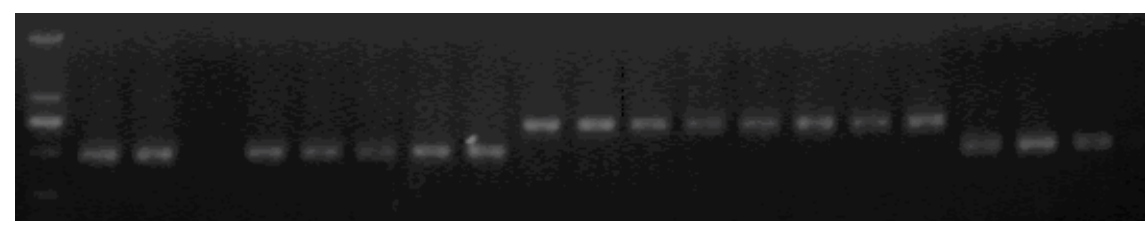

$\begin{array}{llllllllllllllllllll}1 & 2 & 3 & 4 & 5 & 6 & 7 & 8 & 9 & 10 & 11 & 12 & 13 & 14 & 15 & 16 & 17 & 18 & 19 & 20\end{array}$

Lanes: 1: DL2000 marker (2000, 1000, 750, 500, 250 bp); 2-9: pH124-Sep15ORF-hSECIS transformed; 10-17: pH124-wtSep15 transformed; 18-20: pH124-Sep15ORF-chSECIS transformed.

(b)



Lanes: 1: DL2000 marker (2000, 1000, 750, 500 bp); 2-9: pH124-Sep15ORF-hSECIS transformed; 10-13: pH124-Sep15ORF-chSECIS transformed; 14-17: pH124-wtSep15 transformed.

(c)

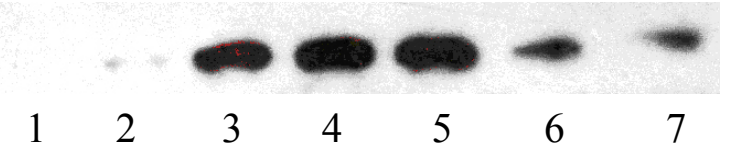

Lanes: 1: wild-type CC-849 cells as a negative control; 2: pH124 empty plasmid transformed as a negative control; 3: pH124-Sep15ORF-hSECIS transformed; 4, 5: pH124-Sep15ORF-chSECIS transformed; 6, 7: pH124-wtSep15 transformed.

RT-PCR was performed to investigate the expression of human Sep15 mRNA in C. reinhardtii, as shown in Figure 2b. Expression of human Sep15 mRNA was detected in six out of eight clones transformed with pH124-Sep15ORF-hSECIS plasmids, or four clones of the pH124-Sep15ORF-chSECIS transformed algae, or three out of four clones of the pH124-wtSep15 transformed algae. 
Those positive clones detected by the genomic PCR and RT-PCR assays were selected for Sep15 protein expression. Sodium selenite was supplemented into the culture medium. Human Sep15 was induced to express in $C$. reinhardtii by heat-shock and light-induction due to the multiple promoters Hsp70-RBCS2. Algal pigment was removed before protein extraction and Western blot analysis. Wild-type $C$. reinhardtii and empty plasmid pH124 transformed $C$. reinhardtii were used as negative controls. As shown in Figure 2c, human Sep15 protein was detected to be expressed successfully in all types of transgenic $C$. reinhardtii cells, but not in two negative controls (Lanes $1 \& 2$ ). Those results showed that all positive transformants, including pH124-wtSep15, pH124-Sep15ORF-hSECIS, and pH124-Sep15ORF-chSECIS, could express human Sep15 in the C. reinhardtii CC-849 cells.

\subsection{Cell Viability of Human Sep15-Transgenic C. reinhardtii}

To compare the cell viability between human Sep15-transgenic and wild-type C. reinhardtii, the same amount of cells of both the transgenic and wild-type algae were inoculated into TAP medium supplemented with sodium selenite. Two types of algal growth curves were plotted according to their time-dependent changes in cell density (Figure 3a,b) and dry weight (Figure 3c,d). The transgenic and wild-type algae showed similar viability when they were cultured without selenite or under low concentrations of selenite $(<10 \mu \mathrm{mol} / \mathrm{L})$. These indicated that the insertion of human Sep15 gene into algal genomes and the expression of exogenous Sep15 proteins in the cells did not affect the viability of $C$. reinhardtii. The transgenic algae grew well and produced human selenoprotein Sep15 when the TAP medium was supplemented with limited amount of selenite. The Se levels of the algae initially increased with the Se concentration in the culture medium $(<10 \mu \mathrm{mol} / \mathrm{L})$, but reached a steady stage for the wild-type or started to decrease for the transgenic at higher concentrations ( $\geq 10 \mu \mathrm{mol} / \mathrm{L})$ (Figure 4). When they were cultured in $5 \mu \mathrm{mol} / \mathrm{L}$ Se medium, the average level of Se was measured as $1100 \mu \mathrm{g} / \mathrm{g}$ dry alga for the transgenic $(n=3)$ and $733 \mu \mathrm{g} / \mathrm{g}$ dry alga for the wild-type $(n=3)$. Although the Se level of transgenic alga was slightly higher than that of wild-type, no significant difference was observed in the present study. Significant inhibition was found in the transgenic alga treated with $15 \mu \mathrm{mol} / \mathrm{L}$ selenite.

Figure 3. Growth curves of human Sep15 transgenic (a, c) and wild-type (b, d) C. reinhardtii.

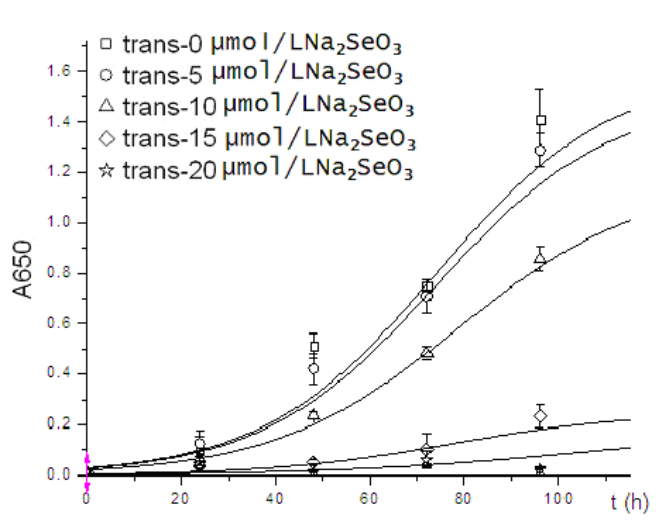

(a)

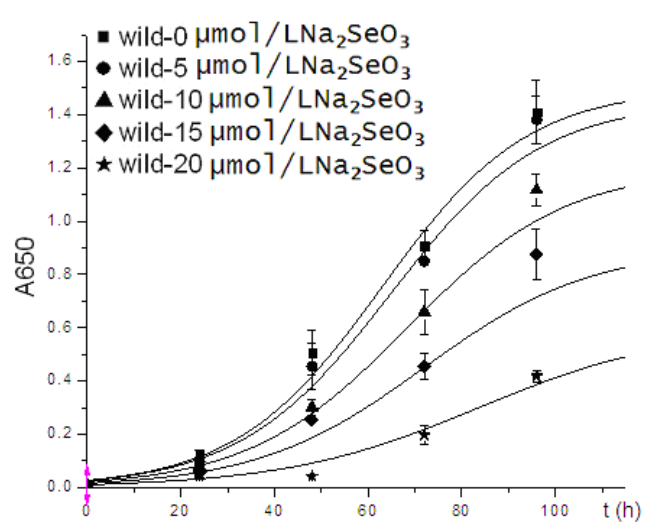

(b) 
Figure 3. Cont.



(c)

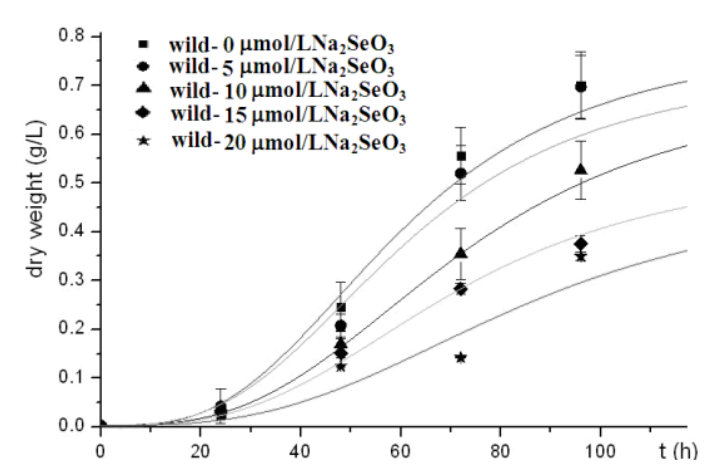

(d)

Figure 4. Se bioaccumulation in the transgenic and wild-type C. reinhardtii.



\section{Discussion}

For a eukaryotic selenoprotein mRNA, a traditional stop codon UGA is located inside its ORF and decoded as a Sec residue under the guidance of an essential SECIS element in the 3'-UTR. Since different SECIS elements from various species may have different efficiency, three types of Sep15 with different SECIS elements were designed and constructed in this paper. As for human Sep15 mRNA its in-frame UGA codon is located in the middle of ORF and two SECIS-like structures are in the 3'-UTR. The first SECIS-like structure does not function, only the second one guides the translation [16]. Therefore, a recombinant Sep15 fragment (Sep15ORF-hSECIS) was constructed to combine human Sep15 OFR directly with the second SECIS element, deleting the first SECIS-like structure in the wild-type. Results in this paper showed that both the wild-type and recombinant transformants could express human Sep15 in C. reinhardtii, which supported the opinion that the translation of UGA to Sec in Sep15 gene is only dependent on the second SECIS element.

C. reinhardtii has been reported to contain ten selenoprotein genes in its genome, except for Sep15 [8]. As SelW1 is one of the two major selenoproteins highly expressed in C. reinhardtii, it is reasonable to deduce that the SelW1 SECIS element has high efficiency in guiding the UGA-to-Sec 
translation. Although each eukaryotic selenoprotein gene has its own specific SECIS element, replacement by other eukaryotic SECIS elements generally does not disturb the decoding of UGA to Sec [17]. In the present study, the SECIS element of $C$. reinhardtii SelW1 was combined with human Sep15 ORF to construct a recombinant of Sep15ORF-chSECIS, in order to test algal SECIS element for human Sep15 expression. The distance between the in-frame TGA and SECIS in the recombinant was designed to resemble that in the $C$. reinhardtii SelW1 gene, in order to reduce distance impact on Sep15 expression efficiency [18]. Results demonstrated that the SECIS elements of both C. reinhardtii SelW1 and human Sep15 were capable of guiding the expression of human Sep15 in C. reinhardtii.

Land plants have lost their ability to synthesize selenoproteins during the evolution process [19]. However, sea plants like $C$. reinhardtii still keep this function to maintain life. The present study makes an effort to express a small selenoprotein in the algal, a new protein expression system. Improvement is in urgent need for getting high yield and expressing other selenoproteins with this platform. Apart from Sep15 expression, the transgenic alga has slightly higher Se level than the wild-type when they were exposed to $5 \mu \mathrm{mol} / \mathrm{L}$ sodium selenite. This level of Se in the transgenic algae is close to that of multiple-step Se-enriched algae (1301 $\mu \mathrm{g} / \mathrm{g}$ dry algae) [20].

Maintaining Se at its physiological level is very important for human health. At least $40 \mu \mathrm{g} / \mathrm{day} \mathrm{Se}$ supplement is essential for adults to maintain the expression and function of selenoproteins, and $300 \mu \mathrm{g} /$ day for cancer treatment [21]. The in vivo function of Se is not only dependent on its level, but also on its form [22,23]. Organic Se generally has low toxicity and high bioavailability compared with inorganic Se. Agronomic biofortification and genetic improvement are potential solutions to increase organic Se in food. Animals, plants and microorganisms grown in Se-rich environment accumulate high level of organic Se $[23,24]$. However, those Se-enriched species can not highly express any specific selenoprotein. Meanwhile, Se binds non-specifically with abundant proteins in those enriched-species [25]. Thus, constructing transgenic species is a good way for improving Se bio-efficiency. $C$. reinhardtii is a non-toxic, edible alga containing many selenoproteins $[9,10]$. The Sep15 transgenic $C$. reinhardtii constructed in this paper improves the nutritional quality of this alga, making it a good and safe Se supplement for human health. It also provides a potential platform for high expression of recombinant selenoprotein in the green alga.

\section{Conclusions}

Three types of human Sep15 gene fragments, including Sep15ORF-hSECIS, Sep15ORF-chSECIS, and wtSep15, were successfully constructed and transformed into C. reinhardtii. All of them were detected to be integrated into the genome of CC-849 cells, and expressed human Sep15 in both mRNA and protein levels. The transgenic algae demonstrated similar growth curves to that of the wild-type when they were cultured in low Se media. Results in this paper indicate that the transgenic C. reinhardtii has potentiality to become a good Se-supplement and a host to express exogenous selenoproteins.

\section{Acknowledgments}

This work was financially supported by the National Natural Science Foundation of China (Grant Nos. 31070731, 30901182, 21271131), the Natural Science Foundation of Guangdong Province 
(No. 10151806001000023), and the Shenzhen Bureau of Science, Technology and Information (No. CXB201005240008A).

\section{Conflict of Interest}

The authors declare no conflict of interest.

\section{References}

1. Jackson, M.I.; Combs, G.F., Jr. Selenium as a cancer preventive agent. In Selenium: Its Molecular Biology and Role in Human Health, 3rd ed.; Hatfield, D.L., Berry, M.J., Gladyshev, V.N., Eds.; Springer Science+Business Media, LLC: New York, NY, USA, 2012; pp. 313-324.

2. Vassort, G.; Turan, B. Protective role of antioxidants in diabetes-induced cardiac dysfunction. Cardiovasc. Toxicol. 2010, 10, 73-86.

3. Neugroschl, J.; Sano, M. Current treatment and recent clinical research in Alzheimer's disease. Mt. Sinai J. Med. 2010, 77, 3-16.

4. Kryukov, G.V.; Castellano, S.; Novoselov, S.V.; Lobanov, A.V.; Zehtab, O.; Guigó, R.; Gladyshev, V.N. Characterization of mammalian selenoproteomes. Science 2003, 300, 1439-1443.

5. Ferguson, A.D.; Labunskyy, V.M.; Fomenko, D.E.; Araç, D.; Chelliah, Y.; Amezcua, C.A.; Rizo, J.; Gladyshev, V.N.; Deisenhofer, J. NMR structures of the selenoproteins Sep15 and SelM reveal redox activity of a new thioredoxin-like family. J. Biol. Chem. 2006, 281, 3536-3543.

6. Jiang, L.; Liu, Q.; Ni, J.Z. In silico identification of the sea squirt selenoproteome. $B M C$ Genomics 2010, 11, 289.

7. Jiang, L.; Ni, J.Z.; Liu, Q. Evolution of selenoproteins in the metazoan. BMC Genomics 2012, $13,446$.

8. Lobanov, A.V.; Fomenko, D.E.; Zhang, Y.; Sengupta, A.; Hatfield, D.; Gladyshev, V.N. Evolutionary dynamics of eukaryotic selenoproteomes: Large selenoproteomes may associate with aquatic life and small with terrestrial life. Genome Biol. 2007, 8, R198.

9. Obata, T.; Shiraiwa, Y. A novel eukaryotic selenoprotein in the haptophyte alga Emiliania huxleyi. J. Biol. Chem. 2005, 280, 18462-18468.

10. Fuhrmann, M.; Oertel, W.; Hegemann, P. A synthetic gene coding for the green fluorescent protein (GFP) is a versatile reporter in Chlamydomonas reinhardtii. Plant J. 1999, 19, 353-361.

11. Novoselov, S.V.; Rao, M.; Onoshko, N.V.; Zhi, H.; Kryukov, G.V.; Xiang, Y.; Weeks, D.P.; Hatfield, D.L.; Gladyshev, V.N. Selenoproteins and selenocysteine insertion system in the model plant cell system, Chlamydomonas reinhardtii. EMBO J. 2002, 21, 3681-3693.

12. He, D.M.; Qian, K.X.; Shen, G.F.; Zhang, Z.F.; Li, Y.N.; Su, Z.L.; Shao, H.B. Recombination and expression of classical swine fever virus (CSFV) structural protein E2 gene in Chlamydomonas reinhardtii chroloplasts. Colloids Surf. B Biointerfaces 2007, 55, 26-30.

13. Wang, C.G.; Hu, Z.L.; Lei, A.P.; Jin, B.H. Biosynthesis of poly-3-hydroxybutyrate (PHB) in the transgenic green alga Chlamydomonas reinhardtii. J. Phycol. 2010, 46, 396-402. 
14. Zhao, Q.B.; Chen, P.; Liu, Q.; Dai, F.Y.; Zhao, P.; Xia, Q.Y. Analysis on the content and distribution of selenium in the silkworm, Bombyx mori by atomic fluorescence spectrometry. Canye Kexue 2007, 33, 482-485.

15. Zhu, Z.J.; Liu, Q.; Chen, P.; Xu, X.; Ni, J.Z.; Yang, S.L.; Song, Y. Seleno-polymannuronate synthesis and resistance to oxidation and apoptosis in Alzheimer's disease cells. Chem. J. Chin. Univ. 2013, doi:10. 7503/cjcu20120462.

16. Berry, M.J.; Banu, L.; Harney, J.W.; Larsen, P.R. Functional characterization of the eukaryotic SECIS elements which direct selenocysteine insertion at UGA codons. EMBO J. 1993, 12, 3315-3322.

17. Martin, G.W.; Harney, J.W.; Berry, M.J. Selenocysteine incorporation in eukaryotes: Insights into mechanism and efficiency from sequence, structure, and spacing proximity studies of the type 1 deiodinase SECIS element. RNA 1996, 2, 171-182.

18. Lobanov, A.V.; Hatfield, D.L.; Gladyshev, V.N. Reduced reliance on the trace element selenium during evolution of mammals. Genome Biol. 2008, 9, R62.

19. Chapple, C.E.; Guigó, R. Relaxation of selective constraints causes independent selenoprotein extinction in insect genomes. PLoS One 2008, 3, e2968.

20. Chen, T.F.; Cui, X.F.; Yang, F.; Zheng, W.J.; Bai, Y. Culture of high Selenium-enriched Spirulina platensis with a stepwise Selenium addition method and its effect on the photosynthetic pigment and protein contents of the microalgae. Food Ferment. Ind. 2005, 31, 48-51.

21. Rayman, M.P.; Infante, H.G.; Sargent, M. Food-chain selenium and human health: Spotlight on speciation. Br. J. Nutr. 2008, 100, 238-253.

22. Rayman, M.P. Food-chain selenium and human health: Emphasis on intake. Br. J. Nutr. 2008, 100, 254-268.

23. Hu, D.; Liu, Q.; Cui, H.; Wang, H.; Han, D.; Xu, H. Effects of amino acids from selenium-rich silkworm pupas on human hepatoma cells. Life Sci. 2005, 77, 2098-2110.

24. Arnault, I.; Auger, J. Seleno-compounds in garlic and onion. J. Chromatogr. A 2006, 1112, 23-30.

25. Liu, Q.; Liang, X.Y.; Hu, D.C.; Chen, P.; Tian, J.; Zhang, H. Purification and characterization of two major selenium-containing proteins in selenium-rich silkworm pupas. Front. Chem. China 2010, 5, 88-98.

(C) 2013 by the authors; licensee MDPI, Basel, Switzerland. This article is an open access article distributed under the terms and conditions of the Creative Commons Attribution license (http://creativecommons.org/licenses/by/3.0/). 